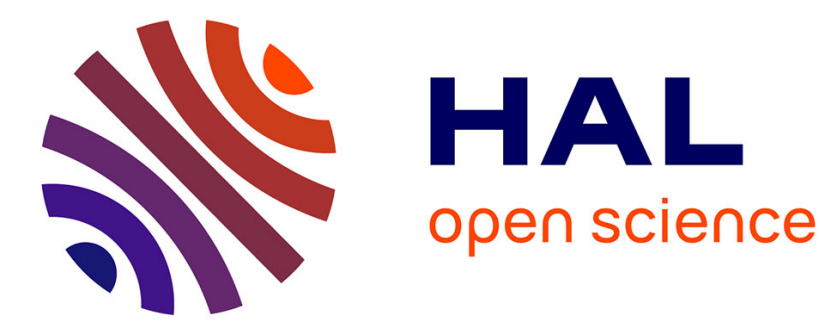

\title{
La raison du complot
}

\author{
Alexandre Rios-Bordes
}

\section{To cite this version:}

Alexandre Rios-Bordes. La raison du complot: Services de renseignement, États-Unis, 1917-1941.

Cultures et conflits, 2017, 2017/4 (108), pp.129-153. 10.4000/conflits.19685 . hal-02497595

\section{HAL Id: hal-02497595 \\ https://hal.science/hal-02497595}

Submitted on 8 May 2020

HAL is a multi-disciplinary open access archive for the deposit and dissemination of scientific research documents, whether they are published or not. The documents may come from teaching and research institutions in France or abroad, or from public or private research centers.
L'archive ouverte pluridisciplinaire HAL, est destinée au dépôt et à la diffusion de documents scientifiques de niveau recherche, publiés ou non, émanant des établissements d'enseignement et de recherche français ou étrangers, des laboratoires publics ou privés. 


\title{
LA RAISON DU COMPLOT
}

Services de renseignement, États-Unis, 1917-1941

\author{
Alexandre Rios-Bordes
}

L'Harmattan | « Cultures \& Conflits »

2017/4 nº 108 | pages 129 à 153

ISSN 1157-996X

Article disponible en ligne à l'adresse :

https://www.cairn.info/revue-cultures-et-conflits-2017-4-page-129.htm

Distribution électronique Cairn.info pour L'Harmattan.

(c) L'Harmattan. Tous droits réservés pour tous pays.

La reproduction ou représentation de cet article, notamment par photocopie, n'est autorisée que dans les limites des conditions générales d'utilisation du site ou, le cas échéant, des conditions générales de la licence souscrite par votre établissement. Toute autre reproduction ou représentation, en tout ou partie, sous quelque forme et de quelque manière que ce soit, est interdite sauf accord préalable et écrit de l'éditeur, en dehors des cas prévus par la législation en vigueur en France. Il est précisé que son stockage dans une base de données est également interdit. 


\section{La raison du complot}

Services de renseignement, États-Unis, 1917-1941

The Logic of Conspiracy. Intelligence Services, United States, 1917-1941

\section{Alexandre Rios-Bordes}

\section{OpenEdition}

Journals

Édition électronique

URL : http://journals.openedition.org/conflits/19685

DOI : $10.4000 /$ conflits. 19685

ISSN : $1777-5345$

Éditeur :

CCLS - Centre d'études sur les conflits lilberté et sécurité, L'Harmattan

Édition imprimée

Date de publication : 31 décembre 2017

Pagination : 129-153

ISBN : 978-2-343-14601-0

ISSN : 1157-996X

Distribution électronique Cairn

\section{CAIRN INFO}

CHERCHER, REPÉRER, AVANCER.

\section{Référence électronique}

Alexandre Rios-Bordes, «La raison du complot », Cultures \& Conflits [En ligne], 108 | hiver 2017, mis en ligne le 31 décembre 2019, consulté le 18 avril 2018. URL : http://journals.openedition.org/ conflits/19685; DOI : 10.4000/conflits.19685 


\title{
La raison du complot
}

\author{
Services de renseignement, États-Unis, 1917-1941 1
}

\section{Alexandre RIOS-BORDES}

Alexandre Rios-Bordes est agrégé et docteur en histoire. Il enseigne l'bistoire contemporaine à l'université Paris Diderot-Paris 7 et il est membre du Laboratoire ICT (EA337). Sa thèse de doctorat a été consacrée à l'émergence de l'"État secret " aux États-Unis au cours du premier XX siècle. Dernière publication : QQuand les services de renseignement repensent la guerre. Eléments d'une archéologie de la "sécurité nationale" (États-Unis, 1919-1941) ", Politix, vol. 27, n 104, 2013, pp. 105-132.

Ct article propose de rompre avec une représentation spontanée des littératures historiques et sociologiques sur le renseignement et la surveillance : l'approche pathologique de la figure du complot. Lorsque confrontés au « complotisme » des professionnels du renseignement intérieur, l'essentiel des travaux ont en effet tendance à en faire le stigmate de dérèglements psychologiques, idéologiques ou bureaucratiques. À partir d'une proposition sous-jacente très rarement explicitée - les complots n'existent pas -, ils ne voient dans cette disposition conspiratoire que le signe de l'usure du sens commun par accoutumance à un soupçon maladif, la conséquence de puissants biais politiques, sociaux et raciaux qui dominent ces institutions fermées, ou encore la résultante de conduites stratégiques conduisant à « gonfler » les menaces pour justifier crédits et effectifs ${ }^{2}$. Nombre d'entre eux laissent volon-

1. Le présent travail a bénéficié du soutien du projet de recherche « Ni guerre, ni paix : les nouages du droit et de la violence dans la transformation des ordres politiques » (ANR-13BSH1-003). L'auteur tient à remercier D. Linhardt et C. Moreau de Bellaing de leurs relectures, ainsi que les évaluateurs anonymes de leurs précieuses et bienveillantes critiques.

2. Si l'on s'en tient au cas qui va nous intéresser, celui des services de renseignement militaires étasuniens au cours du premier XXe siècle : les historiens plus ou moins officiels se bornent à évoquer la visible usure du bon sens (B. W. Bidwell, History of the Military Intelligence Division, Department of the Army General Staff, 1775-1941, Frederick, University Publications of America, 1986 ; Dorwart J. M., Conflict of Duty: the U.S. Navy's Intelligence Dilemma, 1919-1945, Annapolis, Md., Naval Institute Press, 1983 ; dans une moindre mesure : Talbert R., Negative Intelligence: The Army and the American Left, 1917-1941, Jackson Miss., University Press of Mississippi, 1991). Les travaux plus critiques font implicitement du « complotisme » un élément à charge de leur réquisitoire, résultante de préjugés 
tiers entendre que la prégnance du schème conspiratif conduit les institutions sécuritaires sur de fausses pistes, transfigure le citoyen - et singulièrement l'opposant - en ennemi, trahit l'illégitimité de cette méfiance d'État. Ce faisant, ces travaux s'en remettent aux interprétations traditionnelles, psychiatrique et politiste notamment, de l'existence et de la persistance d'explications conspiratoires du social - produits du détraquement, de l'égarement, du mensonge ${ }^{3}$. Ils se conforment aussi au biais normatif qui voudrait notamment que ces services ne considèrent que des menaces «légitimes» de manière « légitime »- lequel biais conduit presque inévitablement à interpréter leurs pratiques en termes d'écarts, de dérives, de déviances ${ }^{4}$.

Cette approche pathologique pose a priori problème. Et ce, avant tout, pour une raison très simple, mais qu'il n'est pas inutile de formuler explicitement comme préalable à la réflexion : quels que soient les facteurs agissant sur leur fonctionnement, quels que soient les intentions et les comportements de leurs agents, et même en laissant un instant de côté l'hypothèse au demeurant fort probable selon laquelle ceux-là cherchent à dire la "vérité », les organes bureaucratiques de ce type sont pris dans une contrainte de vraisemblance. Par là il faut entendre qu'à tous les niveaux de ces bureaucraties, et de manière plus décisive encore à l'interface avec leurs autorités supérieures, s'exerce une pression à transmettre des informations, des interprétations, des analyses pour le moins vraisemblables à court et moyen terme. Dans l'économie de l'information qui règle l'essentiel des relations internes et externes des services de renseignement, l'erreur trop souvent constatée, et à plus forte raison les divagations improbables ou les mensonges, risquent fort, sur la durée, d'entamer sévèrement le crédit de ceux qui les profèrent ${ }^{5}$. Pour se prémunir de ce péril, ces administrations mettent d'ailleurs en place - ont très vite mis en place, dès

politiques, sociaux et raciaux (Kornweibel T., Seeing Red: Federal Campaigns Against Black Militancy, 1919-1925, Bloomington, Indiana University Press, 1998 ; et Investigate Everything: Federal Efforts to Compel Black Loyalty During World War I, Bloomington, Indiana University Press, 2002 ; Bendersky J. W., The "Jerwish Threat": Anti-Semitic Politics of the U.S. Army, New York, Basic Books, 2000), ou preuve de la dérive suspicieuse, voire de l'emprise d'une sorte de paranoïa collective s'emparant de l'institution (Donner F. J., The Age of Surveillance: The Aims and Methods of America's Political Intelligence System, New York, Knopf/Random House, 1980). D'autres entrouvrent enfin la piste bureaucratique, celle de la tendance à chercher à alimenter les échelons supérieurs en matériaux justifiant les efforts engagés (Jensen J., Army Surveillance in America, 1775-1980, New Haven, Yale University Press, 1991).

3. Boltanski L., Énigmes et complots : une enquête à propos d'enquêtes, Paris, Gallimard, 2012, pp. 240-310.

4. Brodeur J.-P., « High Policing and Low Policing : Remarks about the Policing of Political Activities », Social Problems, vol. 30, n 5, 1983 ; et Dobry M., « Le renseignement dans les démocraties occidentales : quelques pistes pour l'identification d'un objet flou ", Cabiers de la sécurité intérieure, $\mathrm{n}^{\circ} 30,1997, \mathrm{pp} .53-85$.

5. Ce qui ne signifie pas que cette économie de l'information se résume à cette question de la vraisemblance. $C f$. Bonelli L., "Un ennemi "anonyme et sans visage" : renseignement, exception et suspicion après le 11 septembre 2001 ", Cultures $E$ conflits, n 58 , été 2005, pp. 101129. En revanche, pas question ici de fabriquer des «faux coupables » pour infléchir le comportement des cibles ou légitimer l'action répressive. Cf. par exemple : Combe S., Une société sous surveillance : les intellectuels et la Stasi, Paris, Albin Michel, 1999 ; Blum A. et Y. 
la période qui va nous concerner ici - de multiples mécanismes devant permettre de s'assurer, à tous les niveaux, du degré de validité de l'information transmise (qualité de la source, crédibilité du contenu, recoupement des données, etc.), donc de discriminer parmi les présomptions, les explications, les récits auxquels officiers et agents sont confrontés.

Dès lors, si l'on veut bien exclure l'hypothèse selon laquelle ces organisations s'enferrent volontairement dans des fabulations possiblement coûteuses, mettent en place des procédures systématiquement défectueuses et persévèrent obstinément dans l'ignorance ou la paranoïa, il faut bien admettre que l'on est confronté à une énigme : pourquoi et comment la conspiration, en tant que schème explicatif des événements politiques et sociaux, pénètre-t-elle l'appareil de sécurité de l'État ? Un questionnement qui ne doit bien entendu rien au hasard, et tout à la conviction que c'est certainement là que le schème conspiratoire peut produire - et, de fait, a produit par le passé - ses effets les plus graves, lorsqu'il s'insinue dans ces « boîtes noires » de l'appareil administratif, s'infiltre dans les mécanismes de production des savoirs de gouvernement, puis qu'à la faveur d'un contexte favorable, ces savoirs sont mobilisés pour informer ou légitimer l'action répressive ${ }^{6}$. Et qu'en conséquence, il y a un enjeu à la fois scientifique et politique à chercher à comprendre les fondements de cette logique, au-delà des interprétations pathologiques dominantes, en tant que figure ordinaire de ce type d'administration.

Le présent article entend montrer que, loin d'être le fruit d'un quelconque dérèglement, l'hypothèse conspiratoire occupe une place tout à fait centrale dans le dispositif du renseignement de sécurité, entendre ici : relatif à l'identification et au suivi des menaces intérieures pouvant peser sur le territoire et la population qui y réside. La démarche suivie implique deux déplacements concomitants par rapport à l'essentiel des travaux existants. Premier déplacement : on n'affronte pas la question du complot de biais, comme une clé permettant d'accéder à autre chose (aux imaginaires politiques et sociaux, aux représentations collectives) ${ }^{7}$, mais plus nettement de front, dans la perspec-

Shapoval, Faux coupables : surveillance, aveux et procès en Ukraine soviétique (1924-1934), Paris, Albin Michel, 2012.

6. Comme ce fut vraisemblablement le cas des fichiers et dossiers du renseignement militaire durant la « chasse aux rouges » des années 1940. Sur cet aspect encore très peu étudié : Jensen J., Army Surveillance in America, op. cit., pp. 232-236 ; Goldstein R. J., Political Repression in Modern America, from 1870 to 1976, Urbana, Illinois University Press, 2001, pp. 299-302.

7. Parmi les «classiques »: Hofstadter R., The Paranoid Style in American Politics, and Other Essays, New York, Vintage Books, 2008, pp. 3-40; Davis D. B. (ed.), The Fear of Conspiracy: Images of Un-American Subversion from the Revolution to the Present, Ithaca, N.Y., Cornell University Press, 1971, pp. 1-22 ; Girardet R., Mythes et mythologies politiques, Paris, Seuil, 1986, pp. 26-62 ; et, plus récemment, les travaux de Timothy Melley (Empire of Conspiracy: The Culture of Paranoia in Postwar America, Ithaca, Cornell University Press, 2000), Peter Knight (Conspiracy Nation: The Politics of Paranoia in Postwar America, New York University Press, 2002) et, en France, de Pierre-André Taguieff (L'imaginaire du complot mondial : aspects d'un mythe moderne, Paris, Mille et une nuits, 2006). Même si d'autres voies ont été frayées, telle celle du complot en tant que stratégie d'action politique (Monier F., Le 
tive de ce que pourrait être une sociologie de la menace ${ }^{8}$. Deuxième déplacement : on prend fermement au sérieux les complots - non pas, bien sûr, pour chercher à en confirmer l'existence, quels qu'ils soient et qu'en soient les auteurs, mais afin de faire l'effort de renouer avec la situation d'indétermination relative qui est celle des acteurs qui se trouvent confrontés à la possibilité de leur existence. C'est à notre sens la seule manière de parvenir, à travers une description précise, à expliquer véritablement leurs comportements individuels et collectifs. Et de contribuer par ce biais à une connaissance des pratiques de contre-renseignement ${ }^{9}$, et singulièrement d'appréhension des menaces, en cherchant à restituer, en-deçà des propriétés sociales des acteurs et des contextes techniques, la forme de rationalité spécifique qui anime ces institutions ${ }^{10}$.

Nous travaillerons pour ce faire à partir d'un cas historique, celui des deux services de renseignement militaire étasuniens, la Military Intelligence Division (MID) et l'Office of Naval Intelligence (ONI), et de la mission " sécuritaire » qu'ils parviennent à s'arroger à partir de la Première Guerre mondiale, puis à conserver au long du quart de siècle suivant. En dépit de leurs faibles moyens, l'un et l'autre sont alors des acteurs majeurs d'un champ bureaucratique de la sécurité intérieure encore émergent et très faiblement structuré. L'un et l'autre sont principalement préoccupés d'une forme spécifique de menace intérieure - la « subversion »-qui justifie à leurs yeux l'institutionnalisation discrète de la surveillance des populations civiles. Nous nous appuyons sur les archives de cette surveillance, librement accessibles aux chercheurs : des milliers de pages de dossiers et de rapports, mais aussi de documents plus ordinaires, d'échanges administratifs plus ou moins formels, et jusqu'à ces « traces du travail administratif » griffonnées en marge des documents qui trahissent parfois les incertitudes, les contradictions, les hésitations ${ }^{11}$. Un matériau dont l'exploitation permet d'accéder au discours et aux pratiques du renseignement de sécurité tel qu'il se configure alors, aux ÉtatsUnis et ailleurs ${ }^{12}$.

Complot dans la Republique : strategies du secret, de Boulanger a la Cagoule, Paris, La Decouverte, 1998), force est de constater que c'est bien cette perspective qui s'est trouvée être considérablement enrichie ces dernières années, en particulier par les historiens. Cf. notamment : Dard O., La Synarchie : le mythe du complot permanent, Paris, Perrin, 2e ed., 2012 ; Tardy J.-N., L'Âge des ombres : complots, conspirations et sociétés secrètes, Paris, Les Belles Lettres, 2015).

8. Cf. Linhardt D., «L'économie du soupçon : une contribution pragmatique à la sociologie de la menace », Genèses, n ${ }^{\circ} 4,2001$, pp. 76-98.

9. Pour reprendre le terme que les anglophones utilisent pour désigner les facettes « défensives » de la discipline (counterintelligence).

10. Bonelli L. et F. Raggazzi, «Low Tech Security: Files, Notes, and Memos as Technologies of Anticipation », Security Dialogue, vol. 45, n 5, 2014, pp. 476-493.

11. Laurens S., «Les agents de l'État face à leur propre pouvoir. Eléments pour une microanalyse des mots griffonnés en marge des décisions officielles », Genèses, n 72, 2008, pp. 26-41; Gayon V., "Écrire, prescrire, proscrire. Notes pour une sociogénétique de l'écrit bureaucratique », Actes de la recherche en sciences sociales, $n^{\circ} 213,2016$, pp. 84-103.

12. Royaume-Uni : Porter B., Plots and Paranoia: A History of Political Espionage in Britain, 
Car ce parti-pris historique, s'il peut certainement se justifier par la difficulté presque insurmontable à s'approcher de ces zones interdites de l'État ${ }^{13}$, s'explique d'abord par l'intuition qu'une démarche généalogique doit permettre de cueillir cette logique conspiratoire au moment où elle émerge, dans les premiers temps de ces institutions singulières, avant qu'un surcroît de prudence n'embrume les discours et que la multiplication des procédures n'étreignent les pratiques (sans toutefois les abolir); et que cette option régressive doit ainsi permettre de déployer des paramètres si profondément ancrés dans le dispositif du renseignement de sécurité qu'ils continuent pour partie d'infléchir les actions de leurs lointains successeurs. C'est cet assemblage complexe qu'il s'agit de décrire, afin de comprendre ce qui amène tendanciellement des institutions organisées, peuplées d'individus a priori raisonnables et toujours soumis à une contrainte de vraisemblance, à percevoir, à supposer, voire à postuler des intentions cachées, des ramifications occultes, des stratégies souterraines, des machinations ourdies dans l'ombre par des têtes pensantes clandestines, et presque toujours une cohérence sous-jacente dans le foisonnement apparemment désordonné des événements politiques et sociaux ${ }^{14}$.

\section{L’impératif de vigilance}

$\mathrm{Au}$ fondement de cette logique conspiratoire, il y a une tension. Il suffit pour le comprendre d'écouter ce que les premiers spécialistes du renseignement disent de leur métier. À quoi sert un service de renseignement ? Au lendemain de l'expérience absolument décisive de la Première Guerre mondiale, qui a vu des services de renseignement militaires étasuniens en état végétatif se hisser en quelques mois au rang d'organes administratifs respectables, leurs responsables identifient sans détour la fonction centrale, et même quintessentielle de leurs organisations : anticiper les menaces. La situation stratégique

1790-1988, Londres, Unwin Hyman, 1989, pp. 12-174 ; Thurlow R., The Secret State: British Internal Security in the Twentieth Century, Oxford, Blackwell, 1994, pp. 15-213; Andrew C., Defend the Realm: The Authorized History of MI5, New York, Alfred A. Knopf, 2009, pp. 29-213 ; Canada : Whitaker R., Kealey G. S. et A. Parnaby, Secret Service: Political Policing in Canada From the Fenians to Fortress America, Toronto, University of Toronto Press, 2012, pp. 60-176. Australie : Cain F., The Origins of Political Surveillance in Australia, Sydney, Angus \& Roberston, pp. 1-106, 229-299. France : Forcade O., La République secrète, Paris, Éditions Nouveaux mots, 2008, pp. 23-31, 40-47, 57-64, 101-131, 363-372, 428-462, 592-615 ; Vidal G., L'armée française et l'ennemi intérieur, 1917-1939 : enjeux stratégiques et culture politique, Rennes, Presses universitaires de Rennes, 2016, pp. 163-219.

13. Même si certains ont su ouvrir des brèches à force de persévérance, une bonne partie des travaux sur la période très contemporaine continuent de reposer, selon les mots très durs du sociologue, "sur des extrapolations à partir de matériaux publiés et de coupures de presse » (Brodeur J. P., The Policing Web, New York, Oxford University Press, 2010, p. 224). Parmi ces «brèches ", on pense en particulier au programme de recherche européen ELISE (European Liberty and Security: Security Issues, Social Cobesion and Institutional Development of the European Union), Final Report, December 2005, pp. 63-64 ; et Bonelli L., «Un ennemi “anonyme et sans visage” », art. cit.

14. Sur la notion de "dispositif »: Dodier N., Barbot J., «La force des dispositifs », Annales. Histoire, Sciences Sociales, vol. 71, n², 2016, pp. 421-450. 
des États-Unis a profondément changé, expliquent-ils en substance : la nation est en situation d'être entrânée à tout moment, fût-ce contre son gré, dans un conflit de grande ampleur. Tenant compte de cet état de fait, il est indispensable de se tenir constamment prêt à un conflit éventuel, ce qui implique non seulement d'accumuler des moyens humains et matériels, mais également d'être en mesure d'identifier ses possibles adversaires, d'évaluer leurs forces, de déterminer leur stratégie et de prévoir leurs agissements - et tout cela non seulement à court terme, pour s'épargner les conséquences désastreuses de l'effet de surprise, mais aussi à long terme, afin d'effectuer les bons choix en matière organisationnelle et stratégique. Toute "armée moderne » se doit de ce fait de disposer de son propre «système d'information », c'est-à-dire d'un service collectant en permanence les données «qui pourraient être utiles en temps de guerre $15 »$. C'est d'abord et avant tout cela, un service de renseignement, et c'est ce qui le rend indispensable aux yeux de ses responsables et promoteurs. «Une armée sans un service de renseignement efficace est comme un boxeur professionnel sans oreilles et sans yeux », résume le chef du renseignement militaire, Marlborough Churchill, au début des années 192016.

Les plus avancés parmi les cadres du renseignement militaire vont toutefois un peu plus loin dans la description : le rôle du service de renseignement n'est pas simplement de collecter des données « utiles », mais de produire des analyses prospectives sur les menaces. "Nous sommes préoccupés du futur », explique le colonel Charles H. Mason, l'un des officiers les plus influents de la MID au sortir du conflit, "le passé et l'instant présent n'ayant d'importance que parce qu'ils permettent de l'élucider ». Mais est-il possible de prévoir le futur avec exactitude ? Selon Mason :

«La réponse est "c'est possible". Et la précision avec laquelle on peut le faire est directement proportionnelle à la quantité d'informations disponibles. Avec des informations complètes, il est possible de déterminer avec une précision totale les situations futures $[\ldots]^{17} »$.

La première exigence du renseignement est donc la recherche de l'exhaustivité, une aspiration qui va concrètement se traduire sous la forme d'injonctions aux échelons inférieurs à « aller au fond des choses » et à transmettre le maximum d'informations, laissant le niveau central trier parmi la masse des données. Deuxième exigence essentielle : la réactivité. «L'estimation minimale

15. Sweeney W. C., Military Intelligence: A New Weapon in War, New York, Frederick A. Stokes Co, 1924, pp. 2-3, 62, 84-85.

16. Churchill W., «MID and How It Works », c. 1921, Yarborough Papers, Military Historical Institute [ci-après : YP MHI], B. 2.

17. Mason C. H., "The Doctrine and Practice of Military Intelligence ", General Staff College, 23 octobre 1919, YP MHI, B. 2. Sauf mention contraire, toutes les traductions sont de l'auteur. 
du futur, poursuit C. H. Mason, doit fournir des informations suffisamment à l'avance pour permettre de se préparer de manière adéquate à faire face aux événements par une action adéquate [sic]. » Là encore, l'impératif se traduit pratiquement par des consignes régulières, invitant les subalternes à ne pas "s'assoir sur les informations ", et à les faire parvenir au plus vite à l'office central 18 .

Enfin et surtout, dans la perspective qui nous préoccupe, le service de renseignement doit se révéler capable de « déterminer les forces et les ressources des ennemis probables ou potentiels [...], leurs objectifs, leurs ambitions et intentions probables, et leurs conduites possibles ", explique à la même époque un autre cadre de la division, le colonel Edwards. Il faut donc ne rien négliger, garder l'esprit ouvert, au besoin faire preuve d'un peu d'imagination, et même ne pas hésiter à « [spéculer] quant aux mobiles, objectifs ou activités futures d'un individu ou d'une organisation 19 ». C'est le troisième précepte essentiel de l'anticipation : l'exigence de spéculation. Ce recours au raisonnement conjectural n'est certainement pas propre aux officiers de renseignement, que l'on peut observer dans d'autres pratiques professionnelles ou situations sociales contemporaines impliquant des formes d'« enquête » au sens large. Il ne s'en exprime pas moins avec une intensité singulière parmi des bureaucrates en uniforme dont la mission spécifique est à leurs yeux d'épargner à leurs institutions et à leur pays, par la production de ce savoir spéculatif, une catastrophe : l'impréparation, la défaite, l'annihilation ${ }^{20}$. La MID est "le représentant de l'ennemi dans nos rangs », confirme C. H. Mason, celui dont le « devoir est de savoir exactement ce que l'ennemi peut faire et a l'intention de faire $21 »$.

«Objectifs », « ambitions », « intentions », « conduites » : l'emploi systématique du pluriel n'est pas anodin. Le futur est par définition ouvert, les «situations » à venir multiples, et avec elles les possibles périls qu'une prudence élémentaire exige de considérer. C'est d'autant plus vrai que la guerre a changé de nature, étendant le spectre de ces menaces potentielles vers l'intérieur du territoire. $\mathrm{La}$ "guerre moderne » est une guerre totale, répète-t-on alors au sein des services. Elle exige la mobilisation de l'ensemble de la nation. Tout élément susceptible de peser sur les «facteurs » militaires et non militaires qui font la « puissance effective» d'une nation relève de ce que certains parmi eux appellent alors l'« équation militaire » et doit en conséquence être

18. Par ex. : Principles of Counter Espionage (\$27), mai 1918, YP MHI, B. 3 ; Instructions for the Organization and Maintenance of the Counter Espionage Service (\$33), aout 1918, YP MHI, B. 3.

19. Nulsen to Lester, ACoS, G2, 1 st Corps Area (“Same letter to All C.A.'s”), 5 juillet 1935, 271A-9 (187), MID Correspondence and Reports, 1917-1941 [ci-après : MID C\&R], B. 356.

20. Aradau C., Van Munster R., Politics of Catastrophe: Genealogies of the Unknown, Londres/New York, Routledge, 2011, pp. 1-16.

21. Mason C. H., «The Doctrine and Practice of Military Intelligence », art. cit. 
l'objet de la vigilance militaire ${ }^{22}$. Et parmi ces éléments domestiques susceptibles d'affecter de l'intérieur la « puissance effective » de la nation, figurent les possibles agissements coordonnés et occultes d'individus hostiles. Cette éventualité n'a rien d'une vue de l'esprit, surtout pas au lendemain d'un conflit durant lequel ces services se sont justement initiés à la " guerre secrète 23 ». C'est la première raison d'être de la présence de l'ONI et de la MID sur le territoire continental étasunien, ces organisations ayant dû faire face à une campagne de sabotage et de déstabilisation orchestrée dans l'ombre par les agents des services de renseignement ennemis. À cette menace primordiale s'en est de surcroît rapidement articulée, puis substituée une autre, dont le projet leur semble tout aussi inquiétant, les tactiques tout aussi sournoises, leurs conséquences tout aussi graves : la subversion révolutionnaire.

L'armistice n'y change rien : ni l'un ni l'autre de ces périls n'ont vocation à s'éteindre avec la fin du conflit à l'hiver 1918 et le reflux de l'agitation sociale intérieure un an plus tard. La guerre secrète a justement pour particularité de se poursuivre en temps de paix, moyen clandestin de la compétition internationale que se livrent les puissances et, le cas échéant, manœuvre préparatoire à un futur conflit ouvert. Quant à la révolution, pour n'avoir pas conquis le monde, elle s'est au moins trouvé une patrie, l'Union soviétique, ainsi qu'une organisation internationale, le Komintern, et peut compter au sein de la société étasunienne sur des partisans qui ne font pas mystère de leurs intentions. Du point de vue des militaires, si la perspective d'une prise du pouvoir reste éloignée, ceux-là sont toujours prêts à profiter de la moindre occasion pour déstabiliser les institutions; et de ce fait sont toujours susceptibles d'être manipulés par des puissances ennemies, en particulier dans la perspective d'un conflit.

« [II] revient ainsi à la branche négative [des services de renseignement] de protéger la nation non seulement de la propagande, de la compétition économique et des agressions politiques habituelles de l'ennemi, mais aussi de ses efforts de guerre visant à créer la dissension et la révolution, à renverser nos institutions, à s'attaquer à nos industries et systèmes de transport par le sabotage, et à nos forces armées par la propagande, l'agitation politique, la limitation des armements ${ }^{24}$, etc. ».

La conclusion s'impose d'elle-même : il ne peut être question, dans ces conditions, de baisser la garde et de cesser d'envisager le pire.

22. Sweeney W. C., Military Intelligence, op. cit., p. 69-74 ; Rios-Bordes A., "Quand les services de renseignement repensent la guerre : éléments d'une archéologie de la "sécurité nationale" (Etats-Unis, 1919-1941) », Politix, vol. 26, n 104, 2013, pp. 105-132.

23. Warner M., "The Kaiser Sows Destruction: Protecting the Homeland the First Time Around ", Studies in Intelligence, vol. 46, n 1, 2002, pp. 3-9.

24. Mason C. H., « The Doctrine and Practice of Military Intelligence », art. cit. 
Au commencement de la logique conspiratoire qui imprègne dès l'origine les services de renseignement, il y a donc un impératif de vigilance. Sans cesser de devoir répondre à l'exigence première qui contraint chacun des échelons de ces structures - et le service lui-même, dans ses rapports avec ses autorités supérieures - à transmettre des informations, des interprétations, des analyses pour le moins vraisemblables, ces mêmes échelons - ces mêmes individus vont devoir répondre à une requête inverse qui consiste, au nom d'une exigence prudentielle, à ne rien écarter a priori, et dont la déclinaison maximale serait une forme de scepticisme renversé consistant à ne rien négliger dont le caractère fictif n'ait pas été démontré. Cet impératif de vigilance constitue, d'une certaine manière, le pôle opposé à la contrainte de vraisemblance, second pôle qui ouvre une tension intellectuelle et pratique au sein de laquelle vont pouvoir se déployer les hypothèses - et les schèmes - conspiratoires. Car, au sein d'un champ des possibles ainsi largement étendu, figure dès l'origine en bonne place la possibilité que les événements s'expliquent par des machinations ourdies dans l'ombre dans l'intérêt de puissances étrangères ou d'organisations hostiles. Voilà le point de départ d'une logique conspiratoire à la fois fondée sur un raisonnement théorique sur l'anticipation et dictée par le constat concret de l'existence de manœuvres clandestines : pour les services de renseignement, le complot est d'abord une possibilité que rien, et certainement pas l'expérience, n'incite à écarter d'emblée.

\section{L'empire de l'abduction}

Mais la bonne logique n'y suffirait pas pour que le complot pénètre si régulièrement et si profondément leur intelligence des événements. Il faut s'intéresser de plus près à ces individus, et faire entrer un deuxième élément en ligne de compte : la socialisation à l'univers professionnel clandestin. Il ne faut pas négliger ce que peut signifier, dès cette période, le fait de franchir les portes des services de renseignement : c'est un basculement, qui passe par la confrontation, puis l'adhésion, l'intériorisation ou, à défaut, la conformité à un ensemble de représentations et de croyances. On y apprend rapidement à penser, à percevoir et à réagir différemment. On s'acclimate à un univers de discours qui véhicule des évidences que la plupart ne questionnent plus vraiment par la suite.

Tous incorporent notamment une sorte d'habitus professionnel dont l'un des éléments essentiels est une vision suspicieuse de la société et du monde, comme invariablement peuplés d'adversaires invisibles organisés pour œuvrer contre les forces armées et, plus largement, contre les institutions, l'ordre, la nation. Bien sûr, cette socialisation se fait à des degrés divers, en fonction des tâches effectuées, des positions occupées, du degré et de la durée d'implication. Mais qu'il opère à l'étranger ou sur le territoire national, qu'il travaille dans un bureau, sur des documents officiels et des articles de presse, ou sur le terrain, sous couverture, parmi ses cibles, l'officier ou agent de renseignement 
doit être à même de discerner, de déceler, au besoin de déterrer, par-delà les apparences, les éléments signifiants, les détails révélateurs, les implications cachées. C'est d'abord cela, être un bon professionnel : ne pas se fier à ce qui semble être, à ce qu'on croit savoir, à ce que les uns et les autres laissent entendre et apercevoir ; être capable de repérer ce qui échappe à «l'observateur moyen » parce que l'on sait être confronté à des ennemis qui dissimulent leurs agissements et travestissent leurs intentions ${ }^{25}$.

C'est un thème récurrent de la littérature de genre et des ouvrages spécialisés dont on recommande la lecture aux réservistes du renseignement militaire : le monde du renseignement est un autre monde, un monde où les événements, les individus et leurs intentions ne sont pas - pas toujours et même presque jamais - ce qu'ils semblent être. On accède ainsi à un autre plan de la réalité, inconnue du citoyen lambda, où se nouent des intrigues, s'échafaudent des stratagèmes et où se jouent et se décident, plus souvent qu'on ne le croit, l'histoire et l'avenir des nations. C'est un univers peuplé d'espions et de «contre-espions » qui se livrent un conflit souterrain, incessant, une guerre "sauvage », qui ne connaît pas de règle et où l'adversaire, qui sait se faire passer pour un autre, «frappe l'ennemi dans le noir, par derrière 26 ». Celui-là peut être au service d'une nation étrangère ; il peut aussi s'incarner, depuis peu, sous une autre forme, celle du révolutionnaire « rouge », qui sait mieux encore que le précédent instrumentaliser les conflits sociaux, utiliser les populations immigrées, manipuler ou infiltrer des groupes annexes ${ }^{27}$.

Ce soupçon instinctif ne s'apprend toutefois pas seulement à la lecture de quelques ouvrages, même dûment recommandés. Le regard se déforme progressivement, à mesure que les semaines et les mois passent, parce que l'on travaille parmi ceux qui savent, que les rapports s'accumulent, que le flot continu des informations se déverse et qu'un nombre suffisamment important d'entre elles se trouvent être corroborées par les événements. Un air de crédibilité conquiert donc doucement les esprits. Dans le même temps, il est très probable qu'un effet « loupe » se mette à jouer, qu'à force de se consacrer à ces questions, de traquer le moindre signe d'activité subversive, on finisse par se convaincre de l'imminence des menaces. Quelques rares officiers sont d'ailleurs assez sensibles à cette possible altération de la perception pour l'évoquer explicitement. En septembre 1921, le commandant Cary C. Crockett, qui

25. «Introduction and Historical Review of Conditions and Agencies tending to create the present tendencies toward radicalism », 10110-1665 (1), MI Reports, R. 15.

26. Cf. Watkins J.B., «Espionage Organization and Practises », 15 janvier 1941, 10039-3007 (119), MID C\&R, B. 2245. Les expressions sont tirées des ouvrages de la bibliographie indicative commentée par Watkins : Tunney T. J., Hollister P. M., Throttled! The Detection of the German and Anarchist Bomb Plotters, Boston, Small, 1919, p. ix ; Baden-Powell R. S., My Adventures as a Spy, Londres, C.A. Pearson ltd., 1915, pp. 9, 61-69 ; Rowan R. W., Spy and Counter-Spy: The Development of Modern Espionage, New York, The Viking Press, 1928, pp. 7-8, 19, 44-48.

27. Hough E., The Web, Chicago, The Reilly \& Lee Co., 1919 (not. pp. 135-136). 
s'apprête à quitter son poste de responsable du renseignement (G2) de la 3 e région militaire livre au chef de la MID, sous la « forme plus libre et plus efficace » d'un simple courrier, son sentiment général sur la situation dans sa zone. Après un portrait des événements locaux qui l'incite à recommander la plus grande prudence, il écrit :

«Il est possible que les peurs exprimées pour la sécurité du public et le bon ordre dans les trois villes mentionnées [Philadelphie, Baltimore, Washington] soient sans fondement et que les rapports sur l'activité radicale soient exagérés. Je suis certain, néanmoins, qu'il y a un très grand nombre d'organisations qui travaillent assidûment de toutes les façons possibles au renversement de notre gouvernement [...]. Bien conscient que l'on a tendance à exagérer l'importance des efforts et des résultats des radicaux après avoir étudié de telles choses pendant un certain temps, j'ai pris garde à cet aspect des choses, et je pense vraiment que ce que j'ai écrit est [un exposé] modéré (conservative) [de la situation] ${ }^{28}$. »

Le commandant Crockett n'évoque certes les possibles effets pervers d'un intérêt prolongé pour les mouvements radicaux que pour mieux les écarter dans son cas. Reste que sa remarque est très révélatrice - et certainement pas sans pertinence.

Ces effets de long terme sont contradictoires. Il est certain qu'à force de s'occuper de ces questions, on acquiert une connaissance très pointue de ses cibles, une capacité à discerner plus vite et mieux, parmi les événements, les profils et les initiatives, ce qui importe de ce qui est insignifiant ou secondaire. Dans le même temps, il est clair que le soupçon systématique devient une sorte de seconde nature qui imprègne et altère l'interprétation des événements et des comportements. L'une des tendances majeures est de refuser a priori que ceux-ci soient le fruit du hasard. Autrement dit : pour la plupart des officiers de renseignement, les coïncidences n'existent pas. Prenons un seul exemple, particulièrement éclairant. Dans son résumé mensuel pour le mois de mars 1941 adressé à la Division du renseignement, le G2 de la 2e région militaire (New York) consacre un court paragraphe aux conflits sociaux qui se multiplient depuis la fin de l'hiver. Il constate qu' " une “coïncidence” veut que les grèves paralysent des usines importantes pour le programme de défense ». Selon lui, dans le contexte international du printemps 1941, il ne s'agit bien entendu nullement d'un coup du sort puisque ces initiatives sont évidemment fomentées «de l'extérieur ». Dès lors, comment s'explique-t-on que ces grèves ne durent pas ou, plus étonnant, qu'il y ait de nombreux endroits où il ne se passe rien ? La réponse est très simple : c'est justement le signe que le pire est probablement à venir.

28. Crockett to Churchill, 20 septembre 1921, 10110-2433 (2), MI Reports, R. 20. 
«L'échec de ces grèves à se matérialiser [dans la 2e région militaire] suggère la possibilité que les leaders syndicaux radicaux alliés aux éléments subversifs planifient, comme cela nous est rapporté, un véritable effort d'envergure, avec la possibilité de grèves générales au moment des grèves projetées dans le métro de New York, et que ces menaces avortées fassent partie d'une "guerre des nerfs" destinée à brouiller les pistes et à faire se relâcher la vigilance avant l'appel à la véritable grosse grève ${ }^{29}$. »

Au sein des services de renseignement, le complot n'est donc pas seulement, on le comprend, une hypothèse que la raison et l'expérience enjoindraient de considérer ; c'est bien souvent une éventualité plausible et même probable, que dicte le flair du professionnel aguerri. Il ne faut pas voir là une dérive. Le renseignement «négatif » est une activité qui repose avant tout sur l'abduction (au sens de Charles S. Pierce), autrement dit : qui implique justement l'exercice de l'intuition, c'est-à-dire le fait d'émettre, à partir de sensations assez vagues, des hypothèses fondées sur des éléments difficilement descriptibles ${ }^{30}$. Et cet instinct professionnel s'acquiert au sein d'un univers qui encourage - et se définit même par - une forme de soupçon systématique, presque ontologique, qui revendique l'idée que le monde n'est pas tel qu'il paraît, que les individus ne sont peut-être pas ceux qu'ils disent être, et que le désordre des événements n'est peut-être qu'apparent ${ }^{31}$. La forme paradigmatique de ce soupçon systématique - ou, si l'on veut, sa conséquence ultime -, c'est la transformation du schème conspiratoire en une sorte de réflexe professionnel. En d'autres termes, pour ces hommes du renseignement, la conspiration possible se fait volontiers probable.

\section{Le chant des signes}

D'autant plus probable que l'œil aiguisé par la suspicion trouve à s'exercer en rencontrant des traces exploitables. Si le renseignement est affaire de conjecture et de flair, il est aussi affaire de diagnostic, c'est-à-dire de déchiffrage de signes indirects permettant d'accéder à des logiques sous-jacentes. En d'autres termes : le renseignement est par excellence une "discipline de l'indice ", selon l'expression autrefois employée par Carlo Ginzburg 32. C’est

29. Monthly Intelligence Summary, $2^{\text {nd }}$ Corps Area, 5 avril 1941, 10110-2662 (374), MI Reports, R. 26.

30. Cf. Linhardt D., «L'économie du soupçon », op. cit., p. 90. Les cadres du service central ne disent pas autre chose lorsqu'ils incitent les échelons inférieurs à ne pas hésiter à faire parvenir, en plus des rapports en bonne et due forme, leurs « observations, commentaires ou impressions qui peuvent être fondées sur de multiples petits détails [...], mais qui sont difficiles à enregistrer ou à expliquer ». Nulsen to Lester, ACoS, G2, 1st Corps Area (“Same letter to All C.A.'s"), 5 juillet 1935, 271-A-9 (187), MID C\&R, B. 356.

31. Une forme de soupçon qui se distingue ainsi du soupçon policier. Cf. Monjardet D., Ce que fait la police : sociologie de la force publique, Paris, La Découverte, 1996, pp. 191-192.

32. Ginzburg C., «Signes, traces, pistes », Le Débat, n 6, 1980, pp. 3-44. 
d'autant plus vrai qu'en dépit des prétentions parfois affichées, les services de renseignement travaillent presque toujours, en raison des multiples difficultés inhérentes à la collecte et au perpétuel manque de moyens, avec une matière première incomplète, et même très réduite; et qu'en conséquence, dès lors qu'il s'agit de donner un sens à des "faits ", quels qu'ils soient, il faut sans cesse combler les vides, c'est-à-dire procéder par approximation, déduire par comparaison, extrapoler à partir d'éléments fragmentaires. Ce « paradigme indiciaire » entraîne deux types de conséquences. Il est certain, d'une part, que le fait de devoir ainsi apprécier des événements, des situations, des comportements fait jouer à plein toutes sortes de préjugés idéologiques, politiques, sociaux et raciaux. Mais ce paradigme de l'indice agit également de manière beaucoup plus profonde, insidieuse, en sécrétant des attitudes analytiques qui affectent constamment la «digestion » des données, et qui jouent subtilement en faveur de la logique conspiratoire ${ }^{33}$.

Première de ces attitudes aisément repérables dans les productions des services : un singulier penchant à faire d'un fait isolé le révélateur d'une réalité beaucoup plus vaste. En dépit des exhortations à recouper les informations, c'est bien souvent que la présence d'un seul « signe » permet de « confirmer ou infirmer une hypothèse existante », ou sert « $d$ 'indicateur d'une tendance en cours $34 »-$ autrement dit : qu'un détail encourage les supputations ou certifie les conclusions. Un bon exemple de cette pente est donné par un rapport de mars 1926 destiné à l'assistant-secrétaire à la Guerre et consacré à l'agitation universitaire à l'encontre du programme de formation des officiers de réserve (ROTC). L'auteur du rapport, le colonel Reeves, se saisit d'un détail à ses yeux très significatif pour dévoiler les véritables origines et les mobiles qui sous-tendent l'effervescence estudiantine : la large circulation, à l'automne précédent, d'une brochure pacifiste intitulée "Military Training in Schools and Colleges of the United States » dont la préface est signée par cinquantehuit individus parmi lesquels nombre «sont très actifs dans le mouvement radical visant à renverser nos institutions ", et dont la publication semble avoir été financée par l'American Fund for Public Service, "plus connu sous le nom de Garland Fund », repère bien connu de «subversifs ». Le lien entre la nébuleuse radicale et l'agitation étudiante est ainsi établi, et c'est assez pour suggérer implicitement la manipulation, et regretter que «malheureusement, il y [a] des centaines de citoyens américains qui s'engagent dans la lutte contre le ROTC sans réaliser la situation radicale qui sous-tend cette attaque 35 ».

33. "Digestion": cf. Mason C. H., "The Doctrine and Practice of Military Intelligence ", art. cit.

"Paradigme de l'indice" : cf. Ginzburg C., "Signes, traces, pistes », art. cit.

34. McCabe E.R.W. «The Military Intelligence Division, WDGS », 1939, Army War College Lectures, MHI.

35. Memorandum for the Assistant Secretary of War, 26 mars 1926, 10314-556 (170), MID C\&R, B. 3026. 
Deuxième attitude analytique très répandue : la propension, en quelque sorte inverse, à déceler des signes à partir d'une réalité sous-jacente dont on postule l'existence. Prenons un exemple situé en fin de période, qui concerne l'une des inquiétudes centrales du renseignement militaire, en particulier en période de mobilisation : l'infiltration communiste des forces armées. En janvier 1941, un mémorandum confidentiel liste les principales tactiques de pénétration employées : le contact direct et individuel, la distribution de littérature ou l'organisation de divertissements.

Mais ce n'est pas tout. D’après le mémorandum,

Le parti communiste recommande à ses agents et organisateurs l'approche des membres des forces armées par l'organisation et la direction de :

Protestations contre le genre, la qualité et la quantité de nourriture et de vêtements ;

Protestations contre l'augmentation du travail et la réduction des privilèges de sortie et de permission;

Protestations contre le coût des frais postaux, de lessive et autres ;

Protestations contre l'isolement et la ségrégation de soldats, et l'absence d'opportunité d'avancement ;

Protestations contre des torts et injustices particuliers dont on peut être victime dans un poste;

Agitation en faveur de l'abolition des cours martiales et du jugement par des jurys d'hommes du rang;

Agitation en faveur de droits et privilèges identiques pour les engagés et les officiers et contre la ségrégation des soldats noirs ;

Agitation en faveur du droit de s'organiser pour dénoncer et corriger les injustices;

Agitation en faveur des droits politiques des membres des forces armées, comme le droit d'adhérer à des partis politique et des syndicats, de voter et d'occuper des fonctions officielles, d'élire leurs propres officiers et contre l'obligation de saluer ;

Agitation pour prévenir l'utilisation de membres des forces armées contre les travailleurs durant les grèves;

Agitation en faveur de l'augmentation de la solde, des pensions spéciales pour les hommes mariés, et pour des primes spéciales pour les parades, etc., en dehors des heures normales de service ;

Agitation en faveur de l'égalité des indemnités des engagés et des officiers ${ }^{36}$.

36. « Reported Methods of Communist Infiltration in the Armed Forces », 18 janvier 1941, 10110-2452 (972), MI Reports, R. 22. 
Si l'on excepte les points 7, 9 et 10, auxquels on peut reconnaître une connotation politique, la liste des revendications et des plaintes est des plus banales. Même si l'on peut certes compter sur la capacité des responsables locaux à faire la part des choses entre les inévitables récriminations des hommes de troupe et une opération de déstabilisation menée par des agents communistes, n'en voilà pas moins ces plaintes revêtues d'une saveur particulière, qui gagnent le statut de possibles symptômes d'une opération subversive.

Troisième tendance particulièrement flagrante : le raisonnement réticulaire. Les officiers et agents de renseignement affichent une propension quasi systématique à chercher - et à trouver - les signes de multiples liens d'un individu à un autre, d'interconnexions d'un groupe à un autre, d'une organisation à une autre et, à partir de là, à en déduire des liens de connivence, de coopération, de coordination. Telle organisation en rassemble d'autres, en particulier celle-ci qui «s'imbrique » dans celle-là, « qui à son tour s'imbrique » dans cette autre, tout cela trahissant l'existence d'un « directoire imbriqué d'organisations ». Les prises de position communes trahissent « l'interdépendance générale de leurs activités », donc la nature véritable des liens ${ }^{37}$, et " la coopération entre des organisations est ordinairement une indication de contrôle centralisé 38 ». Au tournant des années 1920, dans la production des deux services, le National Civil Liberties Burean (NCLB) fait figure de modèle du genre. Tout au long des mois de guerre, affirme un rapport de synthèse consacré à la «situation radicale », l'organisation de défense des objecteurs de conscience, puis des victimes de la répression anti-séditieuse a «étendu ses tentacules » en collaborant avec l'American Union Against Militarism, l'Emergency Peace Federation, le Woman's Peace Party et le Socialist Party, et plus occasionnellement avec le Church Peace Union et l'American Peace Society. Il suffit de surcroît de se pencher sur « les affiliations de certains des plus importants membres de son comité directeur » pour démontrer les multiples liens avec la mouvance radicale et subversive. D'autres courriers et documents permettent de leur associer d'autres noms et d'étendre les ramifications aux milieux révolutionnaires nationalistes, indiens, irlandais ou noirs. La conclusion s'impose d'elle-même : bien que l'on ne puisse le prouver « au sens juridique du terme ", il est évident que le NCLB constitue l'un des nœuds d'un réseau d'organisations travaillant de concert à l'instauration d'un « chaos » politique et social propice à « la réalisation de [leurs] aspirations 39 ».

37. Par exemple: Churchill to Cowles, 14 décembre 1922 ; Cowles to Churchill, 19 décembre 1922, 10110-2491 (1-2), MI Reports, R. 22.

38. Evaluation of Reports from 2nd Corps Area, 14 août 1933, 10110-2662 (60), MI Reports, R. 25.

39. «Introduction and Historical Review of Conditions and Agencies tending to create the present tendencies toward radicalism ", janvier 1920, 10110-1665 (1-102), MI Reports, R. 15. Les expressions entre guillemets sont extraites du document. 
Encouragés à spéculer leur possibilité, enclins à admettre leur existence, les hommes du renseignement perçoivent donc de surcroît de multiples signes d'actions souterraines. S'appuyant nécessairement sur des données très incomplètes, officiers et agents décryptent les événements et les comportements à partir d'éléments qu'ils considèrent être des indices révélant les identités, les activités ou les intentions véritables ${ }^{40}$. Cette dimension indiciaire a pour conséquence de laisser jouer une série d'attitudes analytiques qui œuvrent tendanciellement en faveur de la logique conspiratoire. Parce qu'un seul signe peut suffire à attester la manœuvre; parce que d'autres peuvent suggérer, par concordance, la machination; parce que d'autres encore peuvent dévoiler, de proche en proche, la coordination occulte ; le travail d'interprétation laisse volontiers s'exprimer ce soupçon systématique si essentiel à l'ethos clandestin, en même temps qu'il le conforte en l'alimentant. La dimension indiciaire complète en quelque sorte la dimension intuitive ${ }^{41}$. Le complot, trahi par de multiples traces, affleure aux yeux de ceux qui le traquent, et de probable se fait pour partie visible.

\section{L'ordre des choses}

Que se passe-t-il ensuite, lorsque l'on passe de l'interprétation à la production, c'est-à-dire au cours de la série d'opérations intellectuelles et matérielles qui transforment, selon la distinction consacrée, les informations en renseignement ? Ce pourrait être - et c'est sans aucun doute - le moment privilégié d'expression d'une hésitation, d'un instant d'incrédulité à l'égard de la présomption de complot. Après tout, c'est une chose que d'envisager une hypothèse, d'avoir le sentiment qu'elle est la bonne, d'être persuadé qu'elle est attestée par des indices ; c'en est une autre de la coucher sur le papier, et au besoin d'argumenter en étayant de faits. Mais en allant voir de près ce processus concret de production, on comprend, là encore, que les opérations matérielles vont jouer dans le sens contraire, notamment parce que les instruments bureaucratiques de ces services sont porteurs - et producteurs - d'une interprétation tendanciellement conspiratoire du social.

Peut-être n'est-il pas inutile de dire que cette tendance tient d'abord à la transcription elle-même ${ }^{42}$. Il est certain que le seul passage à l'écrit a tendance à produire une impression de certitude, et à transformer les intuitions en convictions, surtout si elles sont fondées sur les dires d'une « source confidentielle fiable ", attestées par des références à des rapports précédents, barrées du tampon «Secret » 43 . C'est à ce moment-là que les bribes d'informations doi-

40. Ce qu'Alain Dewerpe a appelé «l'herméneutique cryptique ». Dewerpe A., Espion. Une anthropologie du secret d'Etat contemporain, Paris, Gallimard, 1994, pp. 106-107.

41. Linhardt D., "L'économie du soupçon », art. cit., pp. 89-90.

42. Goody J., La Raison graphique : la domestication de la pensée sauvage, Paris, Éditions de Minuit, 1978.

43. "Source confidentielle fiable »: expression utilisée sur les tampons d'évaluation des sources au sein de la MID dans les années 1930. 
vent une première fois être mises en récit, mises en contexte, mises en relation, ce que l'on encourage parfois explicitement en cherchant à contenir le formalisme. C'est encore à ce moment-là que se concrétise le désir des rédacteurs de persuader leurs destinataires de l'exactitude des éléments rapportés et de la pertinence des interprétations proposées. Ce n'est pas tout : les contenus sont ensuite l'objet de constants changements de forme qui épurent encore la présentation des situations et des faits. En clair : la même information fait l'objet de réécritures, de reformulations, de réinterprétations ; et surtout de simplifications, de réductions et de sélections nécessaires à la confection « de résumés, qui à leur tour résument d'autres résumés, de façon à réduire finalement les multiples incidents du monde à une simplicité exploitable 44 ». Au long de ce processus graduel de synthèse, on a inévitablement perdu les éléments de contexte, la perspective fine, les hésitations et les incertitudes au profit de quelques «faits » récurrents bien établis, ce qui a inévitablement pour conséquence de simplifier la présentation, de durcir les raisonnements, de renforcer artificiellement les liens de causalité.

Mais quel que soit l'impact de la transcription, les informations passent surtout au filtre de multiples systèmes de classification de l'information qui véhiculent les éléments-clés d'une vision tendanciellement conspiratoire. Rien de surprenant à cela : ces grilles de lecture sont justement conçues pour ordonner le classement des indices et la présentation des signes. Les plus élaborés de ces systèmes présentent ainsi fort logiquement la menace subversive de manière quasi synoptique, déclinée dans ses différentes tendances principales flanquées des mouvances secondaires et annexes, tandis que toute une série de liens de collaboration ou de subordination entre les différents acteurs est établie et objectivée ${ }^{45}$. Ces liens engendrent ensuite une série de contraintes intellectuelles et pratiques qui contribuent à renforcer un peu plus l'impression de cohérence des événements et des comportements. Le mécanisme est assez simple : aussi fine que soit l'interprétation d'un phénomène, la compréhension d'une situation ou l'évaluation d'une cible, il va bien falloir inclure le rapport dans une catégorie, insérer les données dans l'une des rubriques prédéfinies, désigner les cibles par l'un des vocables consacrés par la nomenclature existante. Et cette contrainte s'exerce d'abord de manière très concrète : il faut bien mettre le rapport dans un dossier, et le dossier dans un tiroir, comme il faut transformer son contenu en fiches et référencer ses éléments essentiels en fonction des mots-clés de l'index ${ }^{46}$. Cela ne signifie certes pas que les offi-

44. Mason C.H., «The Doctrine and Practice of Military Intelligence », art. cit. Voir par exemple les rapports mensuels adressés au chef d'état-major dans la seconde partie des années 1930 : Memorandum for the CoS, 4 novembre 1936 ; Memorandum for the CoS, 3 juillet 1937, 271A-9 (203aa, 207a), MID C\&R, B. 356.

45. Par exemple : «G2 - Index of Digest of Subversive Situation », 7 janvier 1932, 271-A-9 (143), MID C\&R, B. 356.

46. Pour une description de cette opération : "Memorandum on the Mobilization of ONI in the Event of National Emergency », s. d. (c. juin 1919), 21036-2344(A), ONI GC, B. 11. 
ciers soient absolument prisonniers de ces « boîtes » et de ces «tiroirs » ${ }^{47}$. Il n'en reste pas moins que ces classifications domestiquent la retranscription, puis la lecture des faits isolés dans un sens conspiratoire ; conduisent à produire des signes secondaires de l'activité subversive sous des formes agrégées et «objectivées " 48 ; et contribuent à renforcer un peu plus les formes de logiques dominantes dans les services, facilitant grandement les raisonnements réticulaires et les extrapolations à partir d'éléments isolés.

Au travers de ces systèmes, les autorités supérieures génèrent de surcroît des attentes auxquelles les structures ont tendance, d'une façon ou d'une autre, à répondre. Car la production du renseignement est affectée par des comportements stratégiques typiques des organisations hiérarchisées. Une certaine forme de prudence incite ainsi tous les échelons à faire part de tout élément, à envisager toute possibilité de manière à ne pas être pris en défaut. S'ajoute que le principal gage d'activité des différents acteurs est la remontée de renseignements : celui qui ne trouve rien, ne s'inquiète pas, ne remplit pas les « cases » préétablies court le risque d'être considéré inefficace ou inutile. À l'inverse, les subalternes peuvent devancer les attentes, et faire ainsi montre de leur implication, infléchir les décisions de leurs supérieurs, obtenir les moyens et les autorisations qu'ils jugent nécessaires ${ }^{49}$. À un niveau supérieur, les services sont dans une situation similaire. Une partie de leur crédibilité, de leur statut, des fonds qui leur sont alloués, voire la continuation de cette mission intérieure que beaucoup jugent essentielle, dépend de l'ampleur des menaces qu'ils portent à la connaissance de leurs autorités. Ce n'est pas dire que les uns et les autres «inventent » les contenus qu'ils transmettent, mais simplement qu'ils sont subtilement encouragés à ne pas mésestimer le moindre risque, ne pas négliger la moindre piste, à bien remplir les « cases ».

Bref : une fois transcrite sur le papier, saisie par l'écriture, ordonnée et filtrée pour être transmise à l'autorité supérieure sous une forme « exploitable », la multitude des « incidents du monde » a toutes les chances de paraître plus cohérente qu'elle ne l'est sans doute dans l'esprit des premiers observateurs. Il n'y a là nul hasard : la mise en forme et la mise en ordre du renseignement sont justement pensées pour stimuler l'intuition et faire émerger les signes. Possibles, probables, visibles et désormais presque tangibles, ces complots, dont on finit par voir se déployer les ramifications sur des centaines de feuillets où se consigne chaque détail avec cette patiente méticulosité qui font les

47. Cf. Desrosières A., La Politique des grands nombres : bistoire de la raison statistique, Paris, La Découverte, 2000, pp. 289-305 ; Bowker G. C., Star S. L., Sorting Things Out: Classification and Its Consequences, Cambridge, MIT Press, 1999, pp. 1-68.

48. Cf. l'évaluation quantitative des signalements et des rapports par catégorie subversive : Report on Subversive Activities, 4th Corps Area, 20 octobre 1940, 10110-2664 (79), MI Reports, R. 28.

49. Voir par exemple : ACoS, G2, Hawaiian Department to ACoS, G2, 11 décembre 1938, 9679115 (1), MID C\&R, B. 2844. Ou encore : ACoS, G2, 9th Corps Area to ACoS, G2, 14 juin 1939, 294-A-45 (1), RG MID C\&R, B. 832. 
évidences incontestables. D’autant moins contestables qu'elles s'inscrivent dans le temps : même s'ils ne donnent pas lieu à d'immédiates déductions, conjectures et indices garnissent d'innombrables dossiers et enrichissent d'imposants fichiers, lesquels régurgiteront au besoin, un peu plus tard, nantis de ce supplément d'âme que donne le temps qui passe, les matières propres à alimenter d'autres hypothèses conspiratoires 50.

\section{L'économie du soupçon}

Du complot donc, un peu partout dans les productions des services de renseignement militaires. Mais pas n'importe quels complots : il est clair que l'on n'y considère ni n'admet n'importe quel type de conspiration. Cela signifie donc qu'en leur sein, ceux-là éprouvent, d'une manière ou d'une autre, la validité des hypothèses qu'ils formulent, comme des explications qu'on leur soumet ou que les autres échelons proposent. C'est le dernier élément du dispositif : l'épreuve. Un élément essentiel, qui permet de fait à l'institution de maîtriser l'incertitude et le soupçon. Celle-ci se joue toutefois dans des conditions singulières. Parce que les services opèrent à l'abri d'un épais voile de confidentialité et que les avis qu'ils produisent ne donnent généralement lieu à aucune forme d'action tangible ; l'éventualité de voir s'engager une épreuve publique est très limitée. Et parce que l'on reconnaît le caractère anticipatoire, donc spéculatif du savoir produit, l'épreuve de vérité se révèle très largement inadaptée et quoi qu'il en soit repoussée vers un avenir incertain. Les explications conspiratoires sont donc soumises à ce qu'il serait convenu d'appeler une épreuve de crédibilité, qui revient en fait à déterminer non pas ce qui est vrai, ou reconnu comme tel à l'extérieur, mais ce qui est, au sein de ces appareils opaques et clos - et, en fonction de la circulation des contenus, au sein des états-majors, des ministères et, sous certaines conditions, un peu au-delà légitime de soupçonner. Or, bien entendu, la distribution de ce soupçon légitime n’est ni égale ni aléatoire.

Dans le cas qui nous concerne, cette économie du soupçon présente trois caractéristiques principales. Premièrement, c'est une économie politique, entendre : que le degré de crédibilité de l'explication par le complot dépend très clairement du positionnement politique des suspects. C'est l'évidence : les profils « radicaux» - « rouges » au sens large - déclenchent presque systématiquement l'hypothèse conspirative. Une partie de l'explication tient aux représentations sociales des officiers et agents des services : l'hypothèse est d'autant plus immédiatement convoquée que les individus ou les organisations sont a priori perçus avec la plus grande méfiance, considérés, à divers

50. Les expressions sont celles employées par C. H. Mason ( $c f$. n. 44). Voir par exemple le cas du leader syndical Walter Reuther à la veille de la guerre. ACoS, G2, 6 ${ }^{\text {th }}$ Corps Area to ACoS, G2, Subject: «Walter P. Reuther ou Reuter », 7 mai 1941, 10110-2666 (437), MI Reports, R. 31. 
titres, comme ennemis de l'ordre social, du système politique, des institutions qui en sont garantes ${ }^{51}$. Mais il ne faut pas s'y tromper : le critère politique renvoie plus profondément à leur conception de la menace. En dépit de ce que les responsables peuvent régulièrement laisser entendre, l'éventualité d'opérations ourdies par des agents étrangers d'une puissance ennemie reste marginale sur l'essentiel de la période. Les services se préoccupent bien davantage de ceux qu'ils désignent comme les « éléments hostiles ou potentiellement hostiles » dont les agissements sont susceptibles d'affecter « objectivement » ces «facteurs » sur lesquels repose la « puissance effective» du pays - stabilité des institutions gouvernementales, bon fonctionnement de l'appareil productif, soutien du peuple à ses gouvernants et à ses forces armées. Dans cette perspective, les activités politiques dites « radicales » sont tout à la fois conçues comme des éléments de façade d'entreprises conspiratives par ailleurs clandestines ; comme l'une des modalités des offensives subversives, moyen de la déstabilisation à long terme de l'ordre, du système économique, de la confiance populaire ; et/ou comme des conduites instrumentalisables, dans le cadre de manœuvres souterraines, à l'insu de ceux qui s'expriment ou qui agissent 52 . À ces trois titres, les opinions ou les activités politiques « radicales » sont donc autant de possibles indices de quelque chose, et donc fondements d'un soupçon légitime.

À ce facteur absolument déterminant, s'en ajoute un autre, en quelque sorte second : cette économie du soupçon est également institutionnelle. Au sein des services, la suspicion d'intrigue est d'autant plus immédiate que les comportements ou les événements touchent à des domaines particulièrement sensibles pour les forces armées. On ne saurait, là non plus, s’en tenir à un surcroît de sensibilité des hommes en uniforme ou à une tactique de bureaucrates bottés. Cette tendance renvoie directement à une conception de la menace intérieure qui continue, quoi qu'il en soit des impératifs liés à la guerre « moderne », de donner la priorité à la préservation du «facteur militaire ", à la fois première et dernière ligne de défense, principal rempart face aux agressions extérieures et, si nécessaire, ultime garant de l'ordre intérieur. La possible subversion des troupes est de ce fait une hypothèse constamment considérée ; et le fait que le parti communiste cherche à « noyauter» l'armée de terre et la marine fait figure de quasi-certitude, alimentée tout au long de l'entredeux-guerres par une pléthore d'indices ${ }^{53}$. Autre domaine particulièrement propice aux machinations : la livraison des matériels militaires. Les services de renseignement considèrent que, parmi « l'infinité de manières d'attaquer une

51. Sur ce point, voir Rios-Bordes A., «Les précurseurs sombres : l'émergence de l'État secret aux États-Unis, ", thèse d'histoire contemporaine, sous la direction de F. Weil, EHESS, pp. 642-654.

52. Par exemple : "Provisional Instructions for the Operations of the MIS in corps area and departments ", 18 mars 1921, 271-A-9 (7), RG 165 E 65, MID Correspondence and Reports, 1917-1941, Box 356.

53. Parmi de très nombreux exemples : Memorandum for the CoS, 30 mars 1935; Memorandum for the Chief of Division, 30 mars 1935, 10110-2452 (221-222), MID C\&R, B. 2834. 
armée par derrière ", le fait de « provoquer des grèves, le sabotage ou le mécontentement dans n'importe lequel des multiples secteurs industriels dont dépend une armée moderne » est une tactique des plus efficaces ${ }^{54}$. Ils sont donc particulièrement prompts à postuler l'existence d'obscurs manipulateurs à l'origine des conflits sociaux ou à supputer des manœuvres occultes lorsque des secteurs stratégiques sont touchés ${ }^{55}$. Bref : toute activité nuisible aux forces armées - ou se développant dans leurs environs immédiats - est possiblement le signe de quelque chose de grave, légitimant la suspicion d'intrigue.

Enfin, ce soupçon est pris dans une économie de la grandeur. Il est, d'une part, de très vastes et très occultes conspirations, puisées dans les répertoires contemporains, que l'on peut envisager même en l'absence de trace - l'hypothèse se suffisant en quelque sorte à elle-même -, sans toutefois aller beaucoup plus loin. Ainsi le chef par intérim de la branche opération de la MID peut-il écrire, dans une rubrique du rapport annuel de juin 1933 destiné au chef d'état-major de l'armée de terre :

«De temps à autres, on a suggéré l'existence d'une société internationale secrète, disposant de nombreuses filiales, œuvrant à la destruction de la société. On désigne alors les Illuminati et on suggère qu'ils existent toujours. D'autres sources laissent entendre que derrière les divers programmes subversifs se trouve un groupe de Juifs utilisant des non-Juifs ainsi que des membres de leur propre race pour promouvoir leur programme. On ne sait pas si ces groupes existent, mais une image plus claire de la situation subversive émerge dès lors que la possibilité de leur existence est admise à l'arrièreplan ${ }^{56}$. »

De l'autre côté, à l'autre bout du spectre en quelque sorte, les machinations immédiates - campagnes de sabotage, grèves générales, soulèvements armés imminents - sont au contraire considérées avec beaucoup de circonspection, et génèrent en pratique deux types d'attitudes : l'une se manifeste par une hésitation quant à la pertinence de l'interprétation dans son ensemble, qui peut conduire au rejet ; l'autre, la plus courante, consiste à distinguer entre la fiabilité de l'ensemble et celle de certains des détails mentionnés 57 . Très concrètement, on distingue alors d'un trait la partie pertinente, puis l'on griffonne en marge une injonction à procéder aux vérifications nécessaires ${ }^{58}$. Le

54. «Propaganda in its Military and Legal Aspects », Military Intelligence Branch, Executive Division, General Staff, s. d. (c. summer 1918), p. 118, YP MHI, B. 3.

55. Memorandum for Colonel Lester, 24 janvier 1941, 10110-2877 (7), MI Reports, R. 23.

56. Estimate of the Subversive Situation, 1er juin 1933, 211-A-9 (163), MID C\&R, B. 356.

57. Mason C. H., « The Doctrine and Practice of Military Intelligence », art. cit.

58. ACoS, G2, 7th Corps Area to ACoS, G2 (Incl. West to Instructor's Office, Nebraska National Guard, 23 mars 1939; Dudley to West, 23 mars 1939; « Report of March 1, 1939 »), 24 mars 1939, 10110-2667 (84), MI Reports, R. 32. 
complot lui-même s'efface alors pour ne laisser subsister que ces éléments qui constituent autant de possibles indices d'autre chose. Ces attitudes sont là encore intimement liées à l'appréhension de la menace. Si les conspirations les plus vastes ne sont que très rarement considérées plus avant, c'est tout simplement parce que, quel que soit leur degré d'authenticité, elles sont trop éloignées pour jouer un rôle dans les événements qui les concernent ${ }^{59}$. Les intrigues de petite échelle et de courte échéance, qui pourraient justifier une réponse immédiate, sont à l'inverse scrutées de très près, les hypothèses soumises à rude épreuve.

Dans le cas qui nous occupe, l'essentiel se joue, si l'on peut dire, à l'échelle médiane, celle des complots d'envergure, aux ramifications étendues, possiblement étrangères, suffisamment insaisissables pour interdire l'infirmation immédiate tout en étant assez circonscrits, consistants, assurés pour justifier l'inquiétude. Complot allemand au cours des premiers mois de guerre; et surtout complot bolchévique, puis communiste, ourdi depuis Moscou, orchestré par le Komintern et opéré par l'appareil clandestin du parti. À cette échelle, la contrainte de vraisemblance s'exerce, comme en témoignent de rares mais significatives occasions où des officiers osent exprimer leurs doutes par écrit, voire refusent carrément de déchiffrer des séquences d'événements au prisme de l'intrigue « rouge ». La logique conspiratoire l'emporte toutefois très nettement, parce que l'on sait d'expérience de quoi les communistes sont capables, on connaît leur capacité à dissimuler leur action et travestir leurs intentions, on relève constamment les traces de leurs activités subversives ; et que, si nécessaire, la hiérarchie rappelle au bon sens les subalternes myopes ou récalcitrants 60 . Conséquence : tout au long de la période considérée, le complot communiste-révolutionnaire fait office de matrice conspirative à partir de laquelle se déploient la plupart des autres manœuvres ou à laquelle elles s'agrègent.

Les services de renseignement ne spéculent donc pas l'existence de complots tous azimuts. Possibles, probables, visibles, tangibles, ces machinations, à condition d'être crédibles : l'hypothèse conspiratoire n'est convoquée que lorsqu'il est considéré légitime de soupçonner que des événements apparemment disparates ou des comportements extérieurement anodins pourraient résulter d'une action concertée et occulte. «Rouge », prenant pour cible les forces armées, dans le cadre d'une intrigue plus vaste : le contenu du scénario

59. La possibilité d'un « complot juif » international est toutefois prise au sérieux pendant plusieurs mois, au sortir de la guerre, dans le sillage de l'émergence des « Protocoles des sages de Sion » et dans le contexte de la révolution bolchévique. Par exemple : «The Power and Aims of International Jewry ", c. août 1919, 245-1 (1), MID C\&R, B. 305. Cf. Bendersky J. W., The «Jewish Threat », op. cit., pp. 47-74, 121-158, 197-204.

60. Par exemple : Estimate of Subversive Situation, ACoS, G2, 5 th Corps Area to ACoS, G2, 12 avril 1933 ; Evaluation of Reports from the 5th C. A., 14 avril 1933; Estimate of Subversive Situation, ACoS, G2, $5^{\text {th }}$ Corps Area to ACoS, G2, 11 mai 1933 ; Evaluation of Reports from the $5^{\text {th }}$ C. A., 12 mai 1933 ; 10110-2665 (34-35), MI Reports, R. 28. 
conspiratif le plus courant ne surprendra guère le lecteur avisé. L'erreur serait toutefois d'entendre l'orientation de ces présomptions comme une simple translation des représentations sociales des officiers et agents des services. Car si la teneur des hypothèses légitimes n'est certainement pas aléatoire, elle n'est pas pour autant arbitraire. Elle s'articule plus profondément à la physionomie du péril domestique tel que le conçoivent alors ces services : une menace politique, avant tout "subversive », fragilisant à plus ou moins long terme les fondements de la « puissance effective »; un péril d'autant plus inquiétant qu'il est susceptible d'affaiblir directement les moyens de défense armée du territoire ; un danger d'autant plus grand qu'il peut s'insérer, au moins « objectivement », dans une manœuvre de plus grande ampleur, révolutionnaire et étrangère. C'est cette lecture de la menace qui détermine in fine, à chaque étape de la circulation dans ces appareils, le degré de crédibilité de l'hypothèse, c'est-àdire le point d'équilibre momentané entre exigence de spéculation et contrainte de vraisemblance.

Nulle dérive donc, ni dérèglement en soi, dans le fait que les services de renseignement envisagent de manière quasi systématique la possibilité du complot. Au contraire : tout concourt ou presque à l'épanouissement d'une interprétation conspiratoire des événements, des comportements, des phénomènes. Dans ces organisations dont la fonction quintessentielle est de collecter des informations pour anticiper les menaces, le devoir de vigilance dicte de ne rien écarter a priori : on se doit de considérer la possibilité des intrigues, d'autant que l'on sait d'expérience pouvoir être confronté à des adversaires qui dissimulent leurs identités, leurs intentions et leurs actions. Le professionnel du renseignement, c'est justement celui qui a totalement intégré cette donnée, qui a pour habitude et même pour réflexe de « rechercher une vérité positive sciemment obscurcie 61 » son intuition lui permet de transpercer constamment la surface du social en reconnaissant les indices des manœuvres occultes. Quant aux cadres intellectuels et matériels qui structurent concrètement la fabrication et la circulation des savoirs clandestins, ils fonctionnent, dans cette perspective, à la fois comme des moyens de stimulation de la spéculation, des instruments de conduite des interprétations et des générateurs de signes secondaires des manœuvres. L'explication par le complot est ainsi une proposition ordinaire que l'ensemble du dispositif sollicite, encourage, nourrit, conforte même ; et éprouve aussi. Car si le complot est une possibilité, on ne spécule pas sans frein ni sans limite, et certainement pas dans n'importe quelle direction : l'intrigue doit être reconnue crédible au regard des menaces spécifiques identifiées par l'institution ${ }^{62}$. 
Le schème conspiratoire n'en est que plus profondément inscrit, dès l'origine, dans le dispositif du renseignement sécuritaire. Au sein de ces singuliers organes, l'évocation du complot n'est donc réductible ni à l'expression de biais idéologiques, ni à un abandon collectif à une théorie « rassurante » 63 , pas plus qu'aux stratagèmes de bureaucrates avec ou sans uniforme. Pour ces hommes de l'art, la conspiration joue un rôle primordial. Elle est une hypothèse au plein sens du terme, qui participe de leur effort de clarification d'une réalité toujours incertaine ${ }^{64}$. De ce point de vue, elle est d'abord une hypothèse élémentaire, une possibilité qu'il faut bien considérer parmi les possibles. C'est surtout une hypothèse cardinale qui permet d'accéder au plan de la réalité où se déploient les menaces et qui renvoie à la finalité de l'opération : le dévoilement. Elle est enfin, pour cette raison, une hypothèse normative, qui a pour fonction - et pour effet - de modifier plus largement les attitudes de ces «travailleurs du soupçon » dans le sens d'une (re)mise en question de la réalité visible ${ }^{65}$. C'est pourquoi tout le dispositif concourt à la fois à la susciter et à la maîtriser, entretenant cette tension fondamentale qui s'exerce sur chaque échelon de la structure, chaque maillon de la chaîne : entre, d'une part, une exigence de spéculation qui implique de tout considérer de manière à ne rien laisser passer, à ne pas être pris en défaut ; et d'autre part, une contrainte de vraisemblance qui requiert de ne produire que des interprétations pour le moins plausibles (et recevables par les autorités supérieures).

Cette description ne doit pas conduire à nier l'influence des représentations sociales - particulièrement importante dans ces institutions fermées - sur la formulation et l'orientation des scénarios conspiratoires; pas plus d'ailleurs qu'il n'est question d'ignorer le poids des routines institutionnelles ou les possibles usages tactiques des mises en garde alarmistes. Ce sont très certainement ces éléments du dispositif qui peuvent produire le déséquilibre, au point même de changer la nature de l'épreuve. Justement : cette réflexion est sous-tendue par la conviction qu'une meilleure connaissance de ce dispositif intellectuel et pratique peut permettre d'ouvrir et de nourrir un nouvel axe de critique du renseignement sécuritaire contemporain. Une critique qui prenne au sérieux les configurations et les contraintes singulières du contre-renseignement pour pointer notamment les conditions de l'épreuve, et singulièrement la préservation de son caractère non arbitraire. Autrement dit : une critique visant à définir les conditions permettant de s'assurer que l'orientation de la suspicion soit

62. Les déterminants de l'épreuve sont très certainement les plus immédiatement variables du dispositif : qu'un peu de temps passe, que le contexte change, que les contours de la menace évoluent, et la suspicion se portera sur d'autres signes, donc sur d'autres profils; que l'on change d'institution, et c'est la distribution des anxiétés qui se trouve subtilement altérée, et ainsi la nature des indices.

63. Girardet R., Mythes et mythologies politiques, Paris, Seuil, 1986, pp. 54-55.

64. Par référence à Isaac Ben Israël, qui parle des services de renseignements comme d'« institutions de clarification de la réalité » (Philosophie du renseignement : logique et morale de l'espionnage, Paris/Tel Haviv, Éditions de l'éclat, 2004, p. 17).

65. Boltanski L., Énigmes et complots, op. cit., pp. 35-50. 
effectivement et constamment ordonnée à la menace explicitement définie cette définition étant, quant à elle, susceptible d'être discutée dans l'espace public. Une entreprise intellectuelle d'autant plus pressante dans le contexte de renforcement des appareils sécuritaires et de confusion croissante des fonctions judiciaires, policières et « sécuritaires ». 